\title{
Comment
}

Open Access

M. Hariprasad*

\section{Determinant of binary circulant matrices}

https://doi.org/110.1515/spma-2019-0008

Received April 22, 2018; accepted August 8, 2019

Abstract: This article gives a closed-form expression for the determinant of binary circulant matrices.

Keywords: Greatest common divisor, Binary circulant matrix, Circulant matrix determinants, Permutations, Toeplitz determinant

MSC: 11A05, 15B05, 15A15, 05A05

\section{Introduction}

Let $C$ be an adjacency matrix of a full directed cycle (or a permutation matrix of cyclic shift corresponding to $n$ letters) which is expressed as

$$
C=\left[\begin{array}{cccccc}
0 & 1 & 0 & \cdots & 0 & 0 \\
0 & 0 & 1 & 0 & \cdots & 0 \\
0 & 0 & 0 & \ddots & 0 & 0 \\
\vdots & \vdots & \cdots & \cdots & \ddots & 0 \\
0 & 0 & 0 & \cdots & 0 & 1 \\
1 & 0 & 0 & \cdots & 0 & 0
\end{array}\right]_{n \times n} .
$$

Let the matrix $I$ denotes the identity matrix of dimension $n$, and the vector $\mathfrak{e}$ denote the $n \times 1$ vector with all ones. Let $(m, n)$ denote the GCD (Greatest common divisor) of integers $m$ and $n$. Any circulant matrix can be represented as $A=\sum_{k=0}^{n-1} a_{k} C^{k}$. The matrix $C$ has eigenvalues $e^{\frac{2 \pi i t}{n}}$ with an eigenvector $u_{t}$, having entry $u_{t}(r, 1)=\left(e^{\frac{2 \pi i t r}{n}}\right)$ for $0 \leq t<n$ and $0 \leq r<n$.

For a permutation matrix $P$, let $\operatorname{sign}(P)=0$ if it represents even permutation and $\operatorname{sign}(P)=1$ if it represents odd permutation.

In [1] the authors claim that when a circulant matrix has the first row $[a, a, \cdots a, b, b, \cdots b]$ (with $a$ repeated $n-k$ times and $b$ repeated $k$ times) then the determinant is 0 when $n \equiv p \bmod k$ and $(p, k) \neq 1$, otherwise the determinant is $k((n-k)(a / k)+b)(a-b)^{n-1}$. Here this claim is proved positively.

\section{Results}

Theorem 2.1. The binary circulant matrix having the first row $[a, a, \cdots a, b, b, \cdots b]$ (with a repeated $n-k$ times and $b$ repeated $k$ times $)$ has determinant zero when $(n-k, n) \neq 1$ and $((n-k) a+k b)(a-b)^{n-1}$ otherwise.

*Corresponding Author: M. Hariprasad: Department of Computational and Data Sciences, Indian Institute of Science, Bangalore-560012, India, E-mail: mhariprasadkansur@gmail.com 
Proof. The binary circulant matrix having the first row $[a, a, \cdots a, b, b, \cdots b]$ (with $k$ repeated $b \mathrm{~s}$ and $n-k$ repeated $a \mathrm{~s}$ ) is of the form

$$
A=a \sum_{t=0}^{n-k-1} C^{t}+b \sum_{t=n-k}^{n-1} C^{t}
$$

Let us consider two sums

$$
S_{1}=\sum_{t=0}^{n-k-1} C^{t}
$$

and

$$
S_{2}=\sum_{t=n-k}^{n-1} C^{t}
$$

We have

$$
S_{1}(C-I)=C^{n-k}-I
$$

and

$$
\begin{aligned}
S_{2}(C-I) & =C^{n}-C^{n-k} \\
& =I-C^{n-k} .
\end{aligned}
$$

From equations (2) and (4) we have

$$
A(C-I)=(a-b)\left(C^{n-k}-I\right) .
$$

When we multiply by an eigenvector ( $C$ and $A$ share same eigenvectors), which is not $\mathfrak{e}$, denoted by $u_{t}$ (for $1 \leq t<n$ ) with eigenvalue $e^{\frac{2 \pi i t}{n}}$ on both sides of equation (5), we get

$$
A u_{t}=\frac{(a-b)\left(e^{\frac{2 \pi i(n-k) t}{n}}-1\right)}{e^{\frac{2 \pi i t}{n}}-1} u_{t} .
$$

When $(n-k, n)>1$, we have a zero eigenvalue from equation 6 . Hence $\operatorname{det}(A)=0$. Note that we have $A \mathfrak{e}=((n-k) a+k b) \mathfrak{e}$.

So the expression for the determinant when $(n-k, n)=1$ is given by,

$$
\begin{aligned}
\operatorname{det}(A) & =((n-k) a+k b) \prod_{t=1}^{n-1} \frac{(a-b)\left(e^{\frac{2 \pi i(n-k) t}{n}}-1\right)}{e^{\frac{2 \pi i t}{n}}-1} \\
& =((n-k) a+k b)(a-b)^{n-1} .
\end{aligned}
$$

The equation (8) is obtained by noting that when $n-k$ is relatively prime to $n$, the sets $\{(n-k) t \bmod n: 1 \leq$ $t<n\}$ and $\{t: 1 \leq t<n\}$ are equal.

Corollary 2.1.1. When a binary circulant matrix is multiplied by a permutation matrix, represented as $B=$ $A P$, where $A$ has first row $[a, a, \cdots a, b, b, \cdots b]$ (with a repeated $n-k$ times and $b$ repeated $k$ times) then determinant of $B$ is

$$
(-1)^{\operatorname{sign}(P)}((n-k) a+k b)(a-b)^{n-1},
$$

when $(n-k, n)=1$ and zero otherwise.

Proof. Note that $\operatorname{det}(B)=\operatorname{det}(A) \operatorname{det}(P)$. If $P$ is even permutation, then it can be represented as product of even number of flips. With flip having submatrix as $\left[\begin{array}{ll}0 & 1 \\ 1 & 0\end{array}\right]$ and each flip has determinant -1 . So if $P$ is odd permutation $\operatorname{det}(B)=-\operatorname{det}(A)$ else $\operatorname{det}(B)=\operatorname{det}(A)$. 


\section{References}

[1] C. Kravvaritis, Determinant evaluations for binary circulant matrices, Spec. Matrices 2 (2014), 187-199. 